Methods Enzymol. 2009 ; 453: 17-31. doi:10.1016/S0076-6879(08)04002-0.

\title{
Autophagic cell death
}

\author{
Michael J. Lenardo ${ }^{1,{ }^{*}}$, Christina K. McPhee ${ }^{2,3}$, and Li Yu ${ }^{1,4}$ \\ ${ }^{1}$ Laboratory of Immunology, National Institute of Allergy and Infectious Diseases, National \\ Institutes of Health, Bethesda, MD 20892, USA \\ ${ }^{2}$ Department of Cancer Biology, University of Massachusetts Medical School, Worcester, MA \\ 01605, USA
}

${ }^{3}$ Department of Cell Biology and Molecular Genetics, University of Maryland, College Park, MD 20742, USA

\begin{abstract}
In this chapter we discuss methods to study autophagic cell death. A large body of evidence demonstrates that autophagy is a cell survival mechanism in response to starvation. The role of autophagy in cell death, however, has long been controversial. Recently, molecular approaches have provided direct evidence that autophagy contributes to cell death in certain contexts. We begin this chapter by outlining methods to quantify cell death, for example by assaying for cell viability. Next, we discuss methods to measure processes involved in cell death, such as caspase activation and autophagy. Finally, we discuss methods to genetically or chemically perturb autophagy in order to test whether autophagy is required for cell death. Together, these approaches provide a guide to investigate the relationship between autophagy and cell death.
\end{abstract}

\section{Introduction}

Some time after the discovery of autophagy in the 1950s, researchers reported the accumulation of autophagosomes in certain types of dying cells (Hendy and Grasso, 1972). Observations regarding the morphology of dying cells led to the categorization of programmed cell death into 3 types: type I, or apoptotic cell death, type II, or autophagic cell death, and type III, or non-lysosomal cell death (Clarke, 1990; Schweichel and Merker, 1973). Until recently, the hypothesis that cells undergoing type II cell death use their own machinery to "eat themselves to death" remained highly controversial (Levine and Yuan, 2005). Obtaining direct evidence for the involvement of autophagy in cell death proved difficult. On the one hand, a large body of evidence demonstrates that autophagy is a cell survival mechanism in response to starvation (Lum et al., 2005; Onodera and Ohsumi, 2005). On the other hand, the absence of molecular evidence demonstrating a requirement for autophagy in causing type II cell death made it unclear whether autophagy that occurs in conjunction with dying cells contributes to cell death or is simply a cell's failed attempt to rescue itself from a lethal insult. A turning point came in the 1990s with a now-classic study by Ohsumi's group, which ushered in the molecular era for autophagy investigations (Mizushima et al., 1998). Thirty-one autophagy genes $(A T G)$ and many mutants of these genes have been identified in the past decade (summarized in Klionsky, 2007). At the same time, powerful new molecular tools such as RNAi silencing have been utilized in autophagy studies. This has made it feasible to study the molecular role of autophagy in cell death. In 2004, two groups provided evidence that autophagy can contribute to a programmed cell

\footnotetext{
Correspondence: lenardo@nih.gov; Tel: 301-496-6754; FAX: 301-402-8530.

${ }^{4}$ Present address: Department of Biological Science and Biotechnology, Tsinghua University, Beijing 100084, China.
} 
death mechanism (Shimizu et al., 2004; Yu et al., 2004), and, in 2007, Berry and Baehrecke demonstrated in vivo that ATG gene function is required for type II cell death occurring in development (Berry and Baehrecke, 2007).

In this chapter, we attempt to clearly define autophagic cell death and provide experimental approaches for further investigation. During the investigation of cell death, complex cellular effects can emerge. In many cases, dead cells bear the hallmarks of different types of cell death pathways, since multiple cell death pathways can be activated in parallel. Prototypical autophagic cell death has features of necrosis, such as swelling, nucleus and organelle degeneration, and rapid loss of membrane integrity (Yu et al., 2006). There are also examples in which it is believed that apoptosis may be the consequence of autophagy (Espert et al., 2006), which can make it challenging to ascribe cell death to one pathway. Moreover, the categorization of a particular variety of cell death as falling into a single type may actually be misleading because this may mask the complexities of a lethal process that has occurred.

Autophagic cell death, by definition, is a type of cell death which requires autophagy, so three criteria must be met to certify autophagic cell death: 1) autophagy must be induced; 2) the induction of autophagy must be parallel to or precede cell death; and 3) inhibition of autophagy must block cell death. We consider any type of cell death fulfilling these criteria to be autophagic cell death (ACD), even if other mechanisms may also contribute to a cell's demise.

The methods related to the study of cell death can be divided into three groups. First, there are methods to quantify cell death, such as trypan blue or propidium iodide (PI) staining. These methods are based upon the notion that dying cells have altered features, such as the loss of membrane integrity, which can be used to distinguish them from living cells. However, these methods do not offer information about the underlying biochemical events leading to cell death. Second, there are assays that provide evidence of triggering of a specific biochemical pathway. For example, measurements of caspase activation can potentially classify a case of cell death as due to the biochemical pathway leading to apoptosis. Third, there are methods that will reveal the precise genetic pathway leading to cell death. RNAi silencing, for example, or the use of inhibitors or genetic mutants, can be used to identify specific genes involved. So, to classify a particular case of cell death, researchers can extract information using each of these approaches that will lead to a diagnosis. ACD involves both autophagy and cell death and therefore both biochemical processes must be detected together with evidence for causality of the former for the latter. In the following section, we discuss these methods in detail.

\section{Methods to quantify cell death}

\section{Trypan blue staining to detect loss of membrane integrity}

1. Collect $1 \times 10^{6}$ cells and wash once with phosphate buffered saline (PBS). Suspend cells in $1 \mathrm{ml}$ PBS. Transfer $100 \mu \mathrm{l}$ of $0.4 \%$ Trypan blue solution (w/v) (Invitrogen, 15250061) to a tube.

2. Add $100 \mu l$ of the cell suspension (dilution factor $=2$ ) and mix thoroughly.

3. With the cover-slip in place, transfer $10 \mu \mathrm{l}$ of the Trypan blue/cell suspension mixture to each chamber of a hemocytometer. Allow each chamber to fill by capillary action.

4. Count all of the cells in the $1 \mathrm{~mm}$ center square and in the four $1 \mathrm{~mm}$ corner squares. Non-viable cells will stain blue due to loss of membrane integrity; viable cells will exclude dye and remain unstained (white). Keep a separate count of 
viable and non-viable cells. The total number of cells will be determined using the following calculations: cells $/ \mathrm{ml}=$ the average count per reference square $\times$ dilution factor $\times 10^{4}$ (refer to the instructions with the hemocytometer to be certain of the boundaries of the reference square). Count both sides of the hemocytometer and average the cell counts for better accuracy. Variation can be high, so if very accurate counts are required, repeat the procedure and average the cell counts from all determinations. If cell viability is low, count more squares or carry out repeated samplings of the cell suspension until a minimum of 50 cells/corpses, i.e., 50 events, have been quantified.

5. Cell viability $(\%)=$ total number of viable cells (unstained)/total number of cells (stained and unstained) $\times 100$.

\section{NOTES}

1. If cells are exposed to Trypan blue for extended periods of time, viable cells may begin to take up dye.

2. Count cells on the top and left touching the middle line of the perimeter of each square. Do not count cells touching the middle line at the bottom or right sides.

3. Make sure most cells are isolated. If greater than $10 \%$ of the cells appear to be clustered, repeat the entire procedure.

4. The cell number should be between approximately 20 to 50 cells per square. If cell numbers are out of range, adjust to an appropriate dilution factor.

\section{Propidium iodide staining for flow cytometry}

Similar to trypan blue, Propidium iodide (PI) detects the loss of membrane integrity, PI penetrates into dead cells and stain DNA, causing strong emission at $620 \mathrm{~nm}$ which can be easily detected by flow cytometry in the FL-3 channel.

1. Dissolve propidium iodide in buffer comprising $1 \mathrm{X}$ PBS, $\mathrm{pH} 7.5,0.2 \%$ bovine serum albumin, Fraction V (BSA; Invitrogen, 11018041), 0.1\% sodium azide at a concentration of $1 \mu \mathrm{g} / \mathrm{ml}$. Use highly purified BSA for the best results.

2. Collect cells by low speed centrifugation [300×g, $5 \mathrm{~min}]$ and wash once with PBS. After the washing step, resuspend each tube of cells in $2 \mathrm{ml}$ of the PI staining solution and mix well. Let the samples incubate for 5 to 30 minutes. Keep the samples in this solution at $4{ }^{\circ} \mathrm{C}$, protected from light, until the samples are analyzed on the flow cytometer.

3. Analyze with FACSCalibur or similar instrumentation: the cells with low forward scatter (FSC) and high FL3 (channel for PI) are dead cells.

\section{NOTES}

1. When collecting adherent cells, it is important to collect and include the detached cells in the culture medium, as most of the detached cells are the dead cells.

2. Keep the solution tightly closed at $4{ }^{\circ} \mathrm{C}$, protected from light. Discard after 1 month.

\section{Lactate Dehydrogenase (LDH)-Cytotoxicity Assay}

Lactate dehydrogenase (LDH) is a soluble cytosolic enzyme that is released into the culture medium following the loss of membrane integrity that results from cell death. Various companies have developed cytotoxicity assay kits that are based on the quantification of LDH leakage from dead cells. Most assays have a "low control," which is the culture 
medium by itself and a "high control," which contains cells permeabilized by lysis buffer to enable the maximal release of LDH. Most LDH assay kits measure LDH activity using a two-step reaction. In the first step, LDH oxidizes lactate to generate NADH; in the second step, the newly-formed NADH and $\mathrm{H}+$ catalyze the reduction of a substrate to form a highly-colored product. This product can be quantified by changes in optical density (OD). For most kits, reactions and readings are carried out in a 96-well plate for convenience.

The following protocol is from Biovision [K6330400]:

1. Collect cells and wash once with corresponding fresh culture medium; then seed $100 \mu \mathrm{l}$ of cells (with $2-10 \times 10^{4}$ cells per $100 \mu \mathrm{l}$ ) in a 96-well plate as follows:

a. Background Control: $100 \mu \mathrm{l}$ medium per well, in triplicate, without cells.

b. Low Control: $100 \mu \mathrm{l}$ of cells in triplicate wells.

c. High Control: $100 \mu \mathrm{l}$ of cells in triplicate: add $10 \mu \mathrm{l}$ Cell Lysis Solution to each well and mix.

d. Test Sample: $100 \mu 1$ of cells in triplicate: add test substances to each well and mix.

2. Incubate the cells in a tissue culture incubator $\left(5 \% \mathrm{CO}_{2}, 90 \%\right.$ humidity, $\left.37{ }^{\circ} \mathrm{C}\right)$ for the appropriate treatment time determined for the test substance. Gently shake the plate at the end of the incubation to ensure that the LDH is evenly distributed throughout the culture medium.

3. Centrifuge the cells at $250 \times \mathrm{g}$ for $10 \mathrm{~min}$ in a centrifuge fit with a plate carrier.

4. Accurately transfer $10 \mu \mathrm{l} /$ well of the clear medium into the corresponding wells of an optically clear 96-well plate.

5. Add $100 \mu \mathrm{LDH}$ Reaction Mix to each well. Mix and incubate for $30 \mathrm{~min}$ at room temperature.

6. Measure the absorbance of all controls and samples with a plate reader equipped with a $450 \mathrm{~nm}(440 \mathrm{~nm}$ to $490 \mathrm{~nm})$ filter. The reference wavelength should be 650 $\mathrm{nm}$.

7. Cytotoxicity $(\%)=($ Test Sample - Low Control $) /($ High Control - Low Control $) \times$ 100.

\section{NOTES}

1. The background control will measure background signal from the reagents and background LDH in the culture medium serum. The background value should be subtracted from all other values prior to calculating the percent cytotoxicity.

2. The quantity of cells to be used per well depends on the cell type. To optimize the assay, a quick test can be performed by using 2,4 and $8 \times 10^{4}$ cells per well to determine the cell number that you should use. The high control should yield an $\mathrm{OD}_{450 \mathrm{~nm}}$ of approximately 2.0 after 30 min of treatment with $10 \%$ Cell Lysis Solution, whereas the low control should yield an $\mathrm{OD}_{450 \mathrm{~nm}}<0.8$. The reaction time should be set to approximately $30 \mathrm{~min}$.

3. A positive control $(1 \mu 1 \mathrm{LDH})$ can be used to test whether all reagents are working properly to quantify active LDH enzyme. 
4. If the test substances are dissolved in a solvent other than PBS, a solvent control that lacks the testing substrates should be performed by adding the same amount of solvent, in triplicate, without the testing substrates.

\section{Measurement of other cell death-related parameters}

Annexin V staining

Note that an early positive signal with annexin $\mathrm{V}$ staining when membrane integrity is still intact is indicative of apoptosis. This is because phosphatidylserine, which is normally restricted to the inner leaflet of the membrane, is externalized during apoptosis. However at later times, when the membrane has been compromised, loss of lipid asymmetry allows Annexin to bind indiscriminately and it can no longer be considered a selective marker of apoptosis.

1. Collect cells (approximately $5 \times 10^{5}$ to $1 \times 10^{6}$ cells per tube) by slow centrifugation $[300 \times \mathrm{g}, 5 \mathrm{~min}]$ at room temperature.

2. Wash the cells once in $500 \mu l$ of cold $1 \mathrm{X}$ PBS buffer, gently resuspend the cells, and then pellet by centrifugation as in step 1 .

3. For each sample of cells, prepare $120 \mu \mathrm{l}$ of Annexin V incubation reagent by combining the following:

$12 \mu l$ 10X Binding Buffer (100 mM HEPES, pH 7.4, $1.5 \mathrm{M} \mathrm{NaCl}, 50 \mathrm{mM}$

$\mathrm{KCl}, 10 \mathrm{mM} \mathrm{MgCl} 2$ and $18 \mathrm{mM} \mathrm{CaCl}_{2}$ )

$12 \mu l$ propidium iodide powder (for example, Sigma-Aldrich, P4170)

$1.2 \mu 1$ Annexin V-FITC [BD Biosciences, 556419]

$95 \mu$ d distilled $\mathrm{H}_{2} \mathrm{O}$

$120.2 \mu 1$ Total

Keep this cocktail in the dark and on ice.

4. Prepare $440 \mu \mathrm{l}$ of $1 \mathrm{X}$ Binding Buffer per sample to use to dilute the cells after incubation by making a 1:10 dilution of the 10 X Binding Buffer in distilled $\mathrm{H}_{2} \mathrm{O}$.

5. Gently resuspend the washed cells in $100 \mu$ l of the Annexin V incubation reagent prepared in step 3.

6. Incubate in the dark for 15 minutes at room temperature.

7. Add $400 \mu \mathrm{l}$ of $1 \mathrm{X}$ Binding Buffer to each $100 \mu \mathrm{l}$ reaction sample.

8. Examine by flow cytometry within 1 hour for maximal signal. Measure FL3 for PI and FL1 for Annexin V-fluoroscein.

\section{NOTES}

1. The binding of Annexin $\mathrm{V}$ to phosphatidylserine is absolutely dependent upon calcium. Therefore PBS or conventional flow cytometry buffers will not promote binding and should not be used as staining buffers unless $1.8 \mathrm{mM}$ calcium has been added. Chelating agents should be avoided.

2. When using adherent cells, both the supernatant and the attached cells should be collected and examined together. 


\section{Caspase activity assay}

Caspases are cysteine-proteases that hydrolyze target proteins with specific amino acid target sequences. Caspases activation is one of major features of apoptosis, In most cases, caspases activation are consider as biochemical hallmarker of apoptosis, however, caspases activation also occurs in autophagic cell death. Caspase fluorescent assay kits detect caspase activation by assaying for the cleavage of a fluorescent substrate.

1. Collect $2-5 \times 10^{6}$ cells by centrifugation at $250 \times \mathrm{g}$ for 5 minutes. Wash once with cold PBS, add $200 \mu$ l of PEC lysis buffer (20 mM PIPES, pH 7.2, $100 \mathrm{mM}$ $\mathrm{NaCl}, 1 \mathrm{mM}$ EDTA, $10 \mathrm{mM}$ DTT, 0.1\% CHAPS, 10\% sucrose) and mixed thoroughly.

2. Incubate cells on ice for 30 minutes (invert samples several times).

3. Centrifuge samples for 5 minutes at $13,800 \times \mathrm{g}$ and decant the clear supernatant fractions into new tubes to determine caspase activity; discard the pellet fractions. Save an aliquot of each supernatant sample for protein quantification.

4. Prepare N-acetyl-Asp-Glu-Val-Asp-p-Nitroanilide (DEVD-pNA, MBL International, BV-1008-13) stock solution or N-acetyl-Tyr-Val-Ala-Asp-pNitroanilide (YVAD-pNA, MBL International, BV-1104-13 stock solution). The protease reactions are carried out with 100-500 $\mu \mathrm{g}$ of protein extract and 160-200 $\mu \mathrm{M}$ substrate in PEC lysis buffer at a final volume of 50-100 $\mu 1$.

5. Place the reaction mix into a flat-bottomed 96 well microtiter plate and read the absorbance at 405-nm using the kinetics setup of a SpectraMax microtiter plate reader or comparable instrument. Acquire samples every 2-20 seconds and determine initial rates of substrate hydrolysis; ensure that the measured rate of substrate hydrolysis is linear.

\section{NOTES}

1. Brief vortex, quick freeze/thaw cycles, or short sonication with a microtip can improve cell lysis but should be avoided if the solution becomes too viscous due to the breakdown of nuclei and the release of DNA molecules into the supernatant.

2. If the samples cannot be tested immediately, they can be stored at $-70^{\circ} \mathrm{C}$ until measurement.

3. An alternative fluorometric assay can be performed using DEVD- or YVAD-AMC (Calbiochem, 218782-1SET). This gives a 10- to 100-fold increase in sensitivity over the pNA substrates, but requires a more sophisticated instrument for quantification.

\section{TUNEL staining}

Various forms of cell death, especially apoptosis, will result in DNA fragmentation. TUNEL is a method for detecting DNA fragmentation. In a typical TUNEL assay, terminal deoxynucleotidyl transferase (TDT) is used to label nicks in the DNA with Bromodeoxyuridine triphosphate (BrdUTP), BrdUTP then be detected by anti-BrdUTP antibody.

The following protocol is from BD bioscienses (556405)

1. Resuspend the cells by swirling the vials. Remove $1 \mathrm{ml}$ aliquots of the control cell suspensions (approximately $1 \times 106$ cells/ml) and place in $12 \times 75 \mathrm{~mm}$ flow cytometry centrifuge tubes. Centrifuge the control cell suspensions for $5 \mathrm{~min}$ at 300 
$x g$ and remove the $70 \%(\mathrm{v} / \mathrm{v})$ ethanol by aspiration, being careful to not disturb the cell pellet.

2. Resuspend each tube of control cells with $1.0 \mathrm{ml}$ of Wash Buffer (6579AZ) for each tube. Centrifuge as before and remove the supernatant by aspiration.

3. Repeat the Wash Buffer treatment (Step 2).

4. Resuspend each tube of the control cell pellets in $50 \mu 1$ of the DNA Labeling Solution (prepared as described below).

DNA Labeling Solution 1

Reaction Buffer (green cap) $10.00 \mu \mathrm{l}$

TdT Enzyme (yellow cap) $0.75 \mu \mathrm{l}$

Br-dUTP (violet cap) $8.00 \mu \mathrm{l}$

Distilled H2O $32.25 \mu \mathrm{l}$

Total Volume $51.00 \mu \mathrm{l}$

5. Incubate the cells in the DNA Labeling Solution for $60 \mathrm{~min}$ at $37^{\circ} \mathrm{C}$ in a temperature-controlled bath. Shake cells every $15 \mathrm{~min}$ to resuspend.

6. At the end of the incubation time, add $1.0 \mathrm{ml}$ of Rinse Buffer (6583AZ) to each tube and centrifuge each tube at $300 \times g$ for $5 \mathrm{~min}$.

Remove the supernatant by aspiration.

7. Repeat the cell rinsing (as in Step 6) with $1.0 \mathrm{~m} 1$ of the Rinse Buffer, centrifuge, and remove the supernatant by aspiration.

8. Resuspend the cell pellet in $0.1 \mathrm{ml}$ of the Antibody Staining Solution (prepared as described below).

Antibody Staining Solution 1 Assay 5 Assays 10 Assays

FITC-Labeled Anti-BrdU (orange cap) $5.00 \mu 1$

Rinsing Buffer (red cap) $95.00 \mu \mathrm{l}$

Total Volume $100.00 \mu 1$

9. Incubate the cells with the FITC-labeled anti-BrdU Antibody Solution in the dark for $30 \mathrm{~min}$ at RT.

10. Add $0.5 \mathrm{ml}$ of the PI/RNase Staining Buffer (65 $85 \mathrm{~A} \mathrm{Z}$ ) to the tube containing the $0.1 \mathrm{~m} 1$ Antibody Staining Solution.

11. Incubate the cells in the dark for $30 \mathrm{~min}$ at RT.

12. Analyze the cells in PI/RNase Staining Buffer by flow cytometry. Analyze the cells within $3 \mathrm{hr}$ of staining to obtain optimal results.

Cells may begin to deteriorate if left overnight before analysis.

\section{Reactive Oxygen Species (ROS) detection}

Reactive oxygen species (ROS) are by products of the normal metabolism of oxygen. ROS are highly reactive due to the presence of unpaired valence shell electrons. High levels of ROS can cause damage to DNA, lipids, and proteins through oxidation, thus ROS are highly toxic. Accumulation of ROS has been detected during various type of cell death including necrosis and autophagic cell death. 
ROS can be detected by staining with $2^{\prime}, 7^{\prime}$-dichlorodihydrofluorescein diacetate (H2DCFDA). Oxidation of nonfluorescent H2DCFDA converts it to the highly fluorescent $2^{\prime}, 7^{\prime}$-dichlorofluorescein (DCF), which can be detect by the flow cytometer in the FL-1 channel

1. Collect cells by centrifuge with slow speed $(300 \times \mathrm{g}, 5 \mathrm{~min})$. and then wash cells twice with 1X PBS.

2. Stain cells with $25 \mu \mathrm{M} 2^{\prime}, 7^{\prime}$-dichlorodihydrofluorescein diacetate (H2DCFDA, invitrogen, D399) in PBS for 30 minutes.

3. Analyze cells to determine ROS by FACScan. Only analyze live cells (the forward scatter high, side scatter low population) and measure FL1 for ROS.

\section{Detection of Loss of Mitochondrial Membrane Potential by Fluorescent Probe JC-1 staining}

The collapse of the electrochemical gradient across the mitochondrial membrane is an early events of apoptosis and it can be detect by using JC- 1 . The JC-1 dye accumulates in healthy mitochondria and give red fluorescence. However, when the mitochondrial potential collapses during cell death, the JC-1 dye can't accumulated in mitochoindria and therefore remains in the cytoplasm in a monomeric form which emitting green fluorescence, that can be easily detected by cofocal microscopy or flow cytometry.

1. Harvest cells (at least $\left.2 \times 10^{5}\right)$ by sow speed centrifugation $(300 \times \mathrm{g}, 5 \mathrm{~min})$. Bring the total volume up to $1 \mathrm{~mL}$ with corresponding fresh culture medium

2. Stain the cell suspension with $2.5 \mathrm{mg} / \mathrm{mL}$ JC-1 [MBL International, JM-1130-5]. Shake the cell suspension until the dye is well dissolved, giving a uniform redviolet color.

3. Incubate the samples in the dark at room temperature for $15-20$ minutes.

4. Wash twice, centrifuging at $500 \times \mathrm{g}$ for $5 \mathrm{~min}$ with a double volume of PBS.

5. Analyze immediately with the flow cytometer; JC- 1 can be measured using both the FL1 and the FL2 channels.

\section{Methods to measure autophagy}

A wide array of methods can be used to assess autophagy. Morphological hallmarks of autophagy can be observed by transmission electron microscopy (EM) and confocal microscopy. EM remains the "gold standard" for assessing autophagy-the high resolution of EM images allows for the detection of distinguishing physical attributes of autophagosomes, such as the double-membrane structures (see the chapter by Ylä-Anttila in this volume). In addition, engulfed cytosol and organelles can be observed within the double-membrane structure. Another advantage of EM is that it permits the assessment of the signs of other types of cell death, such as nuclear condensation which is characteristic of apoptosis. EM is also the gold standard for diagnosing apoptosis and necrosis as forms of cell death. However, EM can be cumbersome, expensive, and time-consuming. Confocal microscopy, on the other hand, offers a relatively easier method to specifically assess autophagy.

Antibody staining for the autophagosomal marker protein LC3 in fixed cells, as well as live imaging for GFP-tagged LC3, have both been widely used (see the chapter by Kimura in this volume). However, researchers must use caution when overexpressing LC3, as this can create artifacts (Klionsky et al., 2008). Alternatively, autophagy can be assessed by monitoring LC3 processing by western blot. Assessing the involvement of other cell death 
mechanisms (such as caspase activation), also provides a facile means to establish the timeline of biochemical events.

Other chapters in this book provide detailed protocols concerning these methods, so we will not discuss them in detail here.

\section{Methods to establish autophagy as the cause of cell death}

Observing the accumulation of autophagosomes in dying cells does not prove that the death is caused by autophagy - on the contrary, it may represent a survival attempt on the part of the cell. To distinguish whether autophagy is the cause or consequence of cell death, it is necessary to inhibit autophagy and then assess whether or not this blocks cell death. Chemical reagents targeting different steps in autophagy are used as autophagy inhibitors (Table 1). 3-methyladenine (3-MA), a type III PI 3'-kinase inhibitor, and wortmannin, another PI 3 '-kinase inhibitor, inhibit the formation of autophagosomes (Yu et al., 2004). Other inhibitors, such as 1) Bafilomycin $A_{1}$ (Espert et al., 2006), a specific inhibitor of vacuolar-type $\mathrm{H}(+)$-ATPases; 2) hydroxychloroquine (Boya et al., 2005), a lysosomal lumen alkalizer; and 3) pepstatin A (Yu et al., 2006), an inhibitor of acid proteases, have also been used to inhibit complete autophagy. Whereas these drugs cannot inhibit autophagosome formation, they do inhibit autolysosome maturation by preventing degradation of the engulfed cytosolic contents. The inherent problem with any chemical inhibitor is non-specific effects. In addition, most autophagy inhibitors do not directly target autophagy genes. For example, as noted above, 3-MA targets PI 3-kinase, which regulates other pathways in addition to autophagy. Great caution should be taken when interpreting data from chemical inhibitor studies. Whenever possible, molecular approaches should be applied to corroborate the results obtained with chemical inhibitors.

Recently, RNAi silencing for autophagy-essential genes has played a major role in molecularly establishing autophagy as necessary for autophagic cell death (table 2). This approach provides the molecular specificity that chemical reagents lack. However, the precise role of most of the ATG gene products is still not fully understood. In addition, Beclin 1, a gene required for autophagy, regulates functions unrelated to autophagy. As a result, caution must be taken when interpreting data obtained from such experiments. Whenever possible, the expression of multiple ATG genes should be knocked down or otherwise molecularly manipulated to confirm involvement in the death process.

\section{Autophagy genes for RNAi silencing}

Various companies provide RNAi design and synthesis services, as well as transfection protocols. Both lipid-based transfection and electroporation have been used for transfecting RNAi into adherent and suspension cell-types. It is worth noting that for some Atg proteins, a very low level may still be sufficient to allow autophagy to proceed, so it is essential to achieve the highest level RNAi transfection efficiency. A New generation of electroporation methods such as the Amaxa nucleofector (http://www.amaxa.com) offer excellent knock down efficiency. A non-specific RNAi control should always be included in experimental designs, and verification of knockdown efficiency by quantifying RNA or protein levels is essential.

\section{Transfect RNAi by Electroporation (Amaxa nucleofection ${ }^{\mathrm{TM}}$ )}

1. Split one dish of confluent cells onto three large dishes. Allow the cells to grow overnight to achieve $70 \%$ confluence.

2. Use trypsin to release adherent cells and centrifuge at $300 \times \mathrm{g}$ for 5 minutes at $4{ }^{\circ} \mathrm{C}$ and resuspend $1 \times 10^{6}$ cells in $100 \mu$ lappropriate Amaxa transfection buffer 
(choose the appropriate buffer according Amaxa cell line database at http://www.amaxa.com/no_cache/cell-database/).

3. Add RNAi (200 pmol) to this cell mixture and mix gently.

4. Transfer the cell/RNAi mixture into a cuvette.

5. Set up the electroporation program according to the Amaxa database.

6. Remove cells from the cuvette with a pipette and place into $5 \mathrm{ml}$ corresponding culture medium to grow out in one well of 6 well plate.

7. 48 hours after transfection, split cells 1 to 3 into 3 wells of 6 well plates, grow overnight.

8. knock down efficiency should be verified by western blot to measure the decrease in the protein level using cells from one well, and proceeding experiments using another two well.

\section{Conclusion}

Thus far, most methods to quantify cell death and measure cell death-related parameters were developed for measuring apoptosis and necrosis. However, rapid progress in autophagy research has revealed new aspects of autophagic cell death. This has led to cogent methods to measure parameters specific to autophagic cell death. For example, the degradation of cell survival factors such as catalase has the potential to be a parameter specific for autophagic cell death in certain circumstances. Methods described in this chapter have been used successfully in investigation of autophagic cell death. However, given the complicated nature of autophagic cell death, there is not yet a "standard" set of methods that can be used universally. Rather, investigators will have to deploy combinations of different methods tailored to the particular biological question of interest.

\section{Acknowledgments}

This research was supported by the Intramural Research Program of the NIH, NIAID.

\section{References}

Berry DL, Baehrecke EH. Growth arrest and autophagy are required for salivary gland cell degradation in Drosophila. Cell. 2007; 131:1137-1148. [PubMed: 18083103]

Boya P, Gonzalez-Polo RA, Casares N, Perfettini JL, Dessen P, Larochette N, Metivier D, Meley D, Souquere S, Yoshimori T, et al. Inhibition of macroautophagy triggers apoptosis. Mol Cell Biol. 2005; 25:1025-1040. [PubMed: 15657430]

Clarke PG. Developmental cell death: morphological diversity and multiple mechanisms. Anat Embryol (Berl). 1990; 181:195-213. [PubMed: 2186664]

Espert L, Denizot M, Grimaldi M, Robert-Hebmann V, Gay B, Varbanov M, Codogno P, BiardPiechaczyk M. Autophagy is involved in T cell death after binding of HIV-1 envelope proteins to CXCR4. J Clin Invest. 2006; 116:2161-2172. [PubMed: 16886061]

Hendy R, Grasso P. Autophagy in acute liver damage produced in the rat by dimethylnitrosamine. Chem Biol Interact. 1972; 5:401-413. [PubMed: 4652592]

Klionsky DJ. Autophagy: from phenomenology to molecular understanding in less than a decade. Nat Rev Mol Cell Biol. 2007; 8:931-937. [PubMed: 17712358]

Klionsky DJ, Abeliovich H, Agostinis P, Agrawal DK, Aliev G, Askew DS, Baba M, Baehrecke EH, Bahr BA, Ballabio A, et al. Guidelines for the use and interpretation of assays for monitoring autophagy in higher eukaryotes. Autophagy. 2008; 4:151-175. [PubMed: 18188003] 
Levine B, Yuan J. Autophagy in cell death: an innocent convict? J Clin Invest. 2005; 115:2679-2688. [PubMed: 16200202]

Lum JJ, Bauer DE, Kong M, Harris MH, Li C, Lindsten T, Thompson CB. Growth factor regulation of autophagy and cell survival in the absence of apoptosis. Cell. 2005; 120:237-248. [PubMed: 15680329]

Mizushima N, Noda T, Yoshimori T, Tanaka Y, Ishii T, George MD, Klionsky DJ, Ohsumi M, Ohsumi Y. A protein conjugation system essential for autophagy. Nature. 1998; 395:395-398. [PubMed: 9759731]

Onodera J, Ohsumi Y. Autophagy is required for maintenance of amino acid levels and protein synthesis under nitrogen starvation. J Biol Chem. 2005; 280:31582-31586. [PubMed: 16027116]

Schweichel JU, Merker HJ. The morphology of various types of cell death in prenatal tissues. Teratology. 1973; 7:253-266.

Shimizu S, Kanaseki T, Mizushima N, Mizuta T, Arakawa-Kobayashi S, Thompson CB, Tsujimoto Y. Role of Bcl-2 family proteins in a non-apoptotic programmed cell death dependent on autophagy genes. Nature Cell Biology. 2004; 6:1221-1228.

Yu L, Alva A, Su H, Dutt P, Freundt E, Welsh S, Baehrecke EH, Lenardo MJ. Regulation of an $A T G 7-$ beclin 1 program of autophagic cell death by caspase-8. Science. 2004; 304:1500-1502. [PubMed: 15131264]

Yu L, Wan F, Dutta S, Welsh S, Liu Z, Freundt E, Baehrecke EH, Lenardo M. Autophagic programmed cell death by selective catalase degradation. Proc Natl Acad Sci U S A. 2006; 103:4952-4957. [PubMed: 16547133] 
Table 1

Autophagy Inhibitors

\begin{tabular}{|l|l|l|l|}
\hline Name & Mechanism & Solvent & Final concentration \\
\hline 3-methyladenine (3-MA) & PI 3-kinase inhibitor & Water & $10 \mathrm{mM}$ \\
\hline wortmaninn & PI 3-kinase inhibitor & DMSO & $0.1 \mu \mathrm{M}$ \\
\hline Bafilomycin A & Vacuolar-type H(+)-ATPase inhibitor & DMSO & $0.1 \mu \mathrm{M}$ \\
\hline Hydroxychloroquine & Lysosomal lumen alkalizer & Water & $30 \mu \mathrm{g} / \mathrm{ml}$ \\
\hline Pepstatin A & Acid protease inhibitor & DMSO & $1 \mu \mathrm{M}$ \\
\hline
\end{tabular}


Table 2

\begin{tabular}{|l|l|}
\hline Target gene & Accession number (Human) \\
\hline atg5 & BC002699 \\
\hline BECN1 & NM-003766 \\
\hline atg7 & NM-006395 \\
\hline atg 8 & NP-115903 \\
\hline lamp-1 & NM-005561 \\
\hline atg10 & NM-8031482 \\
\hline atg12 & NM-004707 \\
\hline
\end{tabular}

